\title{
Leveraging RSSI for Robotic Repair of Disconnected Wireless Sensor Networks
}

\author{
Kyle A. Luthy and Edward Grant \\ Department of Electrical and Computer \\ Engineering \\ North Carolina State University \\ Raleigh, NC 27695, USA \\ \{kaluthy, egrant\}encsu.edu
}

\author{
Thomas C. Henderson \\ Department of Computer Science \\ University of Utah \\ Salt Lake City, UT 84112, USA \\ tchacs.utah. edu
}

\begin{abstract}
Many recent deployments of environmental sensor networks have focused on obtaining measurements across large and inhospitable areas. With increasing scale it becomes impractical to deploy or maintain such systems by hand. This paper evaluates large scale network disconnectivity and highlights the underlying issues related to the environment and node characteristics. Furthermore, it examines how a low cost and adaptive method of robotic repair can be applied to large area networks using received signal strength measurements for simple navigation and placement.
\end{abstract}

\section{INTRODUCTION}

The benefit of a large area wireless sensor network is that it can instantaneously provide a sensory image of a region. This is particularly important in dynamic, time critical situations such as wildfires where localized information may be insufficient to make behavioral predictions. The wildfire scenario has effectively been demonstrated through real world deployments in both [1] and [2]. Large area deployments are also useful for scientific inquiry into environmental processes which are affected by many factors, not all of which occur locally. This is exemplified by projects such as Glacsweb [3] which studied glacial movements and relayed data over $2.5 \mathrm{~km}$ or the SensorWeb project in Antarctica [4] which sought out biological activity in a $-40^{\circ} \mathrm{C}$ environment. On the other extreme, researchers have also used sensor networks stretching over $3 \mathrm{~km}$ to monitor volcanic activity in Ecuador [5]. All of these deployments highlight concerns related to the integrity of the network's connectivity. These concerns include the asymmetry of radio links [5], variation and losses in connectivity [1][3], and all are concerned with ground interference, consequently elevating at least some nodes to improve connectivity at distance [1][2][3][4][5]. To address these concerns and improve reliability all of these deployments were placed by hand.

As nodes become cheaper, larger numbers of nodes will be used for deployments. Eventually they will grow to the point where hand placement is not practical and in many cases, unsafe. In these less precise deployments, the level of reliability cannot be assured and will require physical adjustments or additions to the network to improve the connectivity. This paper addresses this issue by introducing an adaptable method for robotic repair of disconnected networks. This is accomplished using simple mobile nodes applying basic rules to received signal strength intensity measurements taken from the network.

The rest of this paper is organized as follows. Section II will further motivate the need for connectivity repair in large area networks through assessment of factors affecting connectivity. Section III will provide an overview of related work. Section IV will discuss the design of the proposed system while Section $\mathrm{V}$ will present preliminary experimentation. Section VI will close with a discussion of results and the direction of future work.

\section{Motivation}

The most likely form of deployment of a large scale wireless sensor network in a remote, inaccessible, or inhospitable area is by air drop. In order to address the connectivity of the network it is important to understand how it is affected by both this type of physical deployment and the inherent difficulties associated with wireless communications. Among the issues involved are:

1) environmental effects

2) radiation pattern of nodes

3) randomness of deployment

4) temporal effects (i.e. node death/birth, movement)

The environment plays a critical role in any wireless system. Obstacles can result in radio shadowing, absorption, scattering, or reflection which can all be detrimental to a wireless link. Line of sight is particularly important with the typical node radio frequency residing between $868 \mathrm{MHz}$ and $2.4 \mathrm{GHz}$. This can be caused by both physical obstacles such as trees and rocks as examined in [6] or by more varying effects such as weather as demonstrated in [7] which reports significant degradation in performance in rain and fog conditions. Even obstacles not lying directly in the line of sight of two nodes can reduce the strength of the link if they lie within the Fresnel zone between the two endpoints. For this very reason, many experiments are performed with the node off of the ground [7]. It is interesting to note that in [1] distances of up to $393 \mathrm{~m}$ were observed given that the quoted range of the mica2 motes used in this deployment is only $300 \mathrm{~m}$ with line of sight. This improvement was attributed to the reduction in obstacles encroaching in the Fresnel zone due to topographic variation in the deployment zone. Elevation changes may also have a beneficial effect as 
mica motes have been observed to transmit better in ground to sky communications [8].

This last point implies anisotropic radiation patterns for mote nodes. Nodes are not typically equipped with high quality antennas since cost is one of their more attractive and enabling characteristics. Anisotropic radiation patterns can result in asymmetric links which can cause difficulty with neighbor discovery and routing protocols since node A can hear node $\mathrm{B}$ but not vice versa implying the importance of relative orientation of nodes. These effects are characterized and empirically studied in [9], [10], and [11]. Another factor resulting in link asymmetry is variation in hardware from node to node introduced in manufacturing. This is discussed in [9] which includes such variation in the proposed Radio Irregularity Model (RIM) which serves as an alternative to the traditional spherical model. Interesting link behavior was also reported in [5] where one node favored a more distant node over its intended neighbor. Holes in radio coverage have also been demonstrated in our laboratory experiments where with a single broadcasting node emitting a count the message did not elicit an LED event on near neighbor nodes but did on further nodes in the same path. Switching the node with another demonstrated the same effect.

Problems associated with the asymmetric communication links are compounded by the randomness of the deployment. When air dropped, nodes will not likely land in an ideal orientation. Furthermore such deployments are largely random and nodes can find themselves out of range, in dense vegetation or in valleys, impeding network connectivity. Although not preferred, this will be one of the few practical methods to deploy large area networks.

Once a deployment is made there is always the possibility of change over time, particularly in disaster conditions. The most obvious consideration is node death. Node death can be the result of hardware failure, battery life, or destruction due to fire, vehicle, stampede, etc. Even if a node is not disabled it can still be removed from communication. In many cases there may have been changes in the environmental topology, such as in an avalanche, or the node itself may have moved. Of course changes in topology and node movement can also result in node birth or discovery where the node is introduced to previously unknown nodes.

Given these issues there is not a high likelihood of total connectivity allowing for routing across the region of interest. This can be countered however by increasing the density of nodes within the region. The more nodes, the more likely to achieve a higher level of connectivity, but at an increased cost. To assess how connectivity levels vary with different densities the following simulation scenario is considered:

Different numbers of nodes are randomly placed within a 1 square kilometer area. Each disconnected network is then counted. This is done barring all of the previously discussed effects except deployment which is considered to be totally random. The radio range of each simulated node has a radius of $100 \mathrm{~m}$ and is isotropic.
Fig. 1 shows a deployment of 25 nodes. Each connected sub network is signified by a matching letter. The circle around it is the assumed $100 \mathrm{~m}$ range. Note that the largest network, 'c', consists of only 4 nodes placed near the extent of their range and two of the subnets (' $\mathrm{l}$ ' and ' $\mathrm{k}$ ') consist of nodes that are almost coincident. No redundancy is exhibited in any of these networks

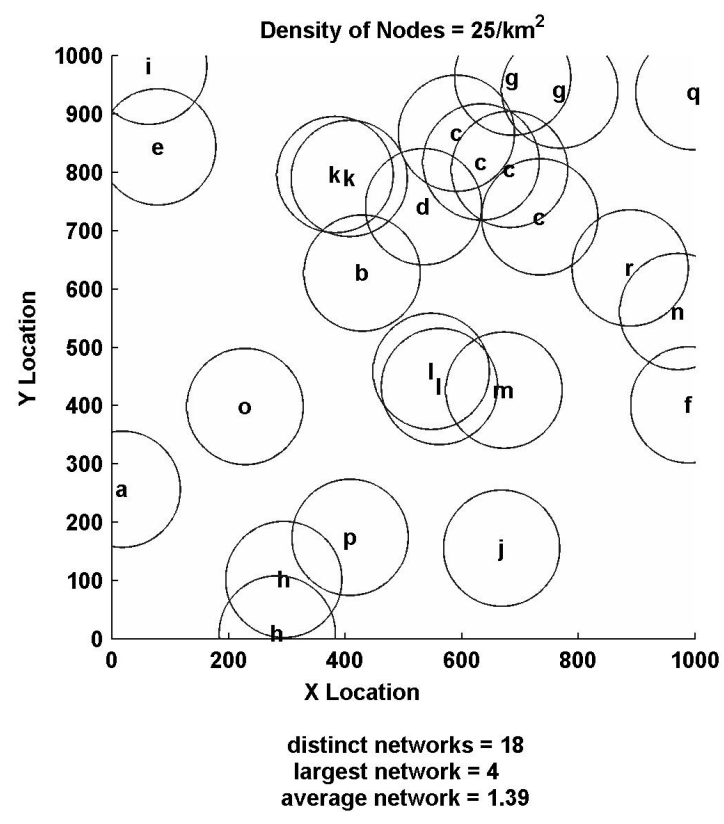

Fig. 1. Connectivity with nodal density of 25 nodes $/ \mathrm{km}^{2}$

Increasing the density of nodes to $100 / \mathrm{km}^{2}$ yields a more manageable scenario as depicted in Fig. 2. Here the largest network, 'b', consists of 31 nodes. There are several large networks that stretch an appreciable distance although some of the more populated networks such as ' $\mathrm{k}$ ' demonstrate redundancy in a confined area. It is important to note that a few additional nodes strategically placed would greatly increase the size of and area encompassed by a single network. Single nodes could connect 'b' to 'd', 'b' to 'c', 'b' to 'e', or 'd' to 'c' for example. Just 5 nodes could greatly increase the sprawl of the network.

With a nodal density of $250 / \mathrm{km}^{2}$ total connectivity is practically achieved with the largest network consisting of 248 nodes. This results in a network with almost total radio coverage. Each population ranging in density from 1 to 250 nodes was simulated 100 times and the following metrics were recorded. First is the average network size, Fig. 3. This metric should be considered with care as it is misleading if used alone to determine connectivity since with densities like $250 / \mathrm{km}^{2}$ there are only 2 networks with a gross disparity in population. This is demonstrated in Fig. 4 which shows the average size of the largest network. It is also useful to consider the number of disjoint networks (Fig. 5) in order to realize the impact of a density on the overall connectivity. The trade-off portrayed in these graphs implies that more nodes may get the job done, but it will only do so at the expense of hardware cost and complexity of communication 


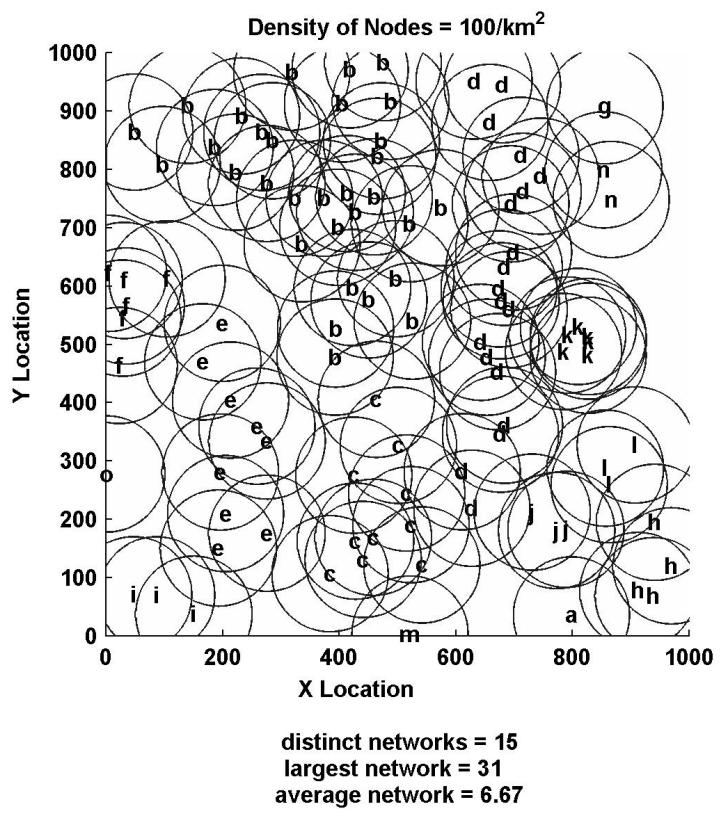

Fig. 2. Connectivity with nodal density of 100 nodes $/ \mathrm{km}^{2}$

protocols. For instance, in Fig. 5 there is not much gain in connectivity between 150 and 250 nodes but the average size of each network as shown in Fig. 3 is 3 times as large. This signifies increased redundancy in the system with a small improvement in connectivity. Fig. 5 is also particularly useful in that it provides a general metric of how many links will need to be established in a deployment of a given density. This will be useful when the time comes to determine how many nodes are required to repair the network. A more probabalistic examination of network connectivity using percolation theory is addressed in [12]. They seek to find the critical density of nodes required to ensure connectivity for any given connection probability function.

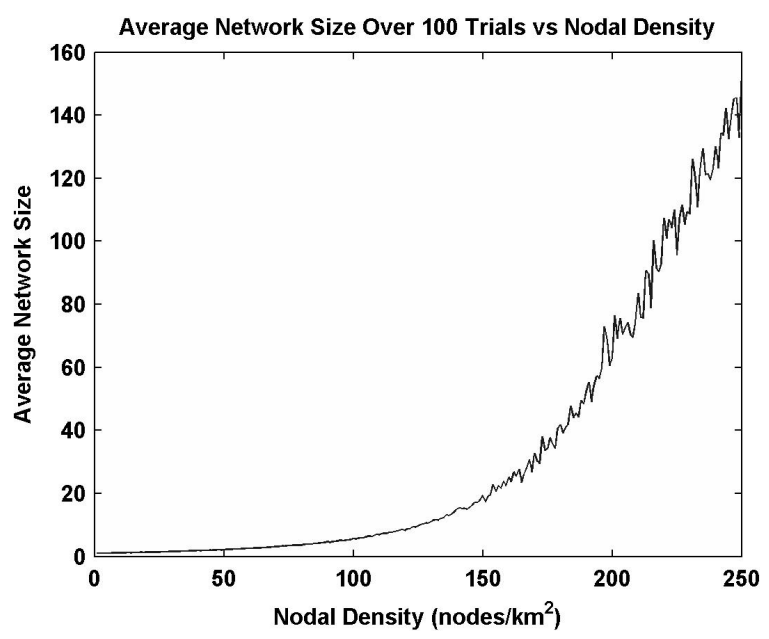

Fig. 3. Average size of networks for varying nodal densities

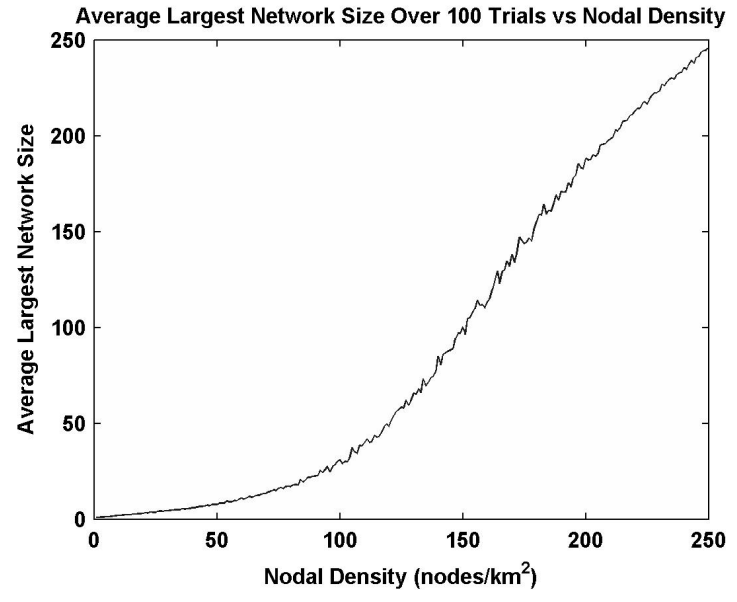

Fig. 4. Average size of the largest network for varying nodal densities

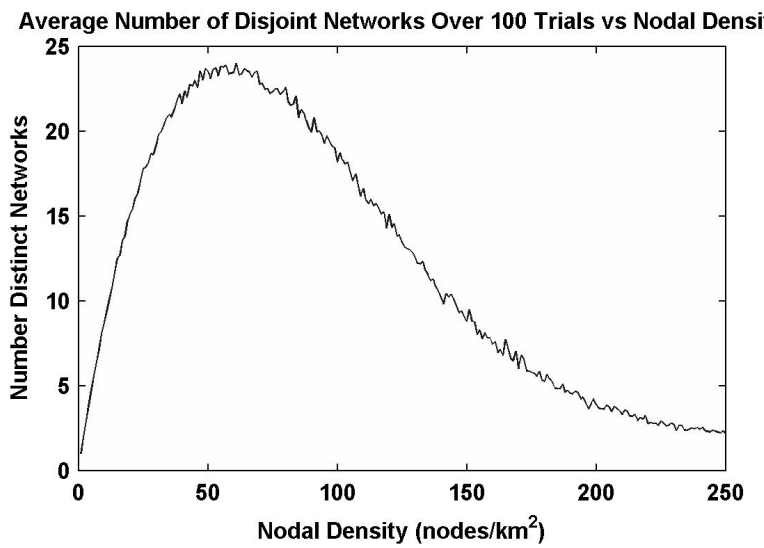

Fig. 5. Average number of disjoint networks for varying nodal densities

\section{RELATED WORK}

Researchers have anticipated some of these problems and have already sought to address them through robotic deployment and repair. Corke et al. have examined both repair and deployment of sensor networks using an autonomous helicopter [8]. This system communicates with deployed nodes via an onboard mote and places new nodes with the aid of a DGPS system. This work also highlights connectivity troubles associated with node orientation, obstacles, and link asymmetry.

Consideration should also be given to autonomous indoor deployments. Although generally deployed across a smaller area, they still must address a large subset of the issues faced in larger, outdoor deployments. Parker et al. [13] use sophisticated leader and helper robots to guide less capable sensor node robots to their deployment points. Sensor nodes are tracked and placed by the helper robots using visual cues and laser ranging information.

Howard et al. [14] apply potential fields to the deployment problem. Interestingly there is no communication necessary for their deployment method, their robots relying only on cues obtained from on board sensor systems. Potential fields are also employed in [15] with more focus on power and 
routing optimization of the resulting network.

The system of [16] proposes deployment of a sensor network by utilizing different broadcast powers for node-tonode and node-to-robot communication. The node-to-robot communication range is the lesser of the two. Therefore when the robot has lost communication with one node, it can place another node that will still be connected to the network by the increased node-to-node power. Each node and robot is equipped with a compass allowing the network to tell the robot where it has not been in order to expand the network.

\section{SySTEM DESCRIPTION}

When developing a robotic system for large area sensor network repair, it is important to optimize cost as well as performance. One of the objectives of using robotic repair is to reduce the cost by deploying fewer nodes. Therefore the cost of the robotic system should not exceed that of simply deploying nodes densely enough to assume connectivity. After all, when deploying in hazardous environments it may be assumed that the robot is an expendable asset.

In most of the previously discussed systems, cost was not a guiding factor. Many of them utilize vision systems, laser range finders, or DGPS. While effective, most people would be reluctant to drop dozens of such systems into a wildfire environment. In order to attain cost effectiveness, the proposed system relies only on a mobile node with no sensory extension other than what is required of the deployed network (temperature sensors, gas sensors, etc.). It operates solely on communication with other nodes (stationary and mobile) and associated RSSI measurements for guidance.

\section{A. Physical Platform}

The robotic platform currently used achieves mobility using a robotic differential drive toy base controlled with a simple driver circuit [17]. While not particularly suited for large scale implementations, it serves its purpose in this proof of concept. As shown in Fig. 6 the base circuitry is attached directly to a tmote-sky module from Moteiv [18]. The mote provides PWM outputs to the base for speed and direction control. Other than the cost of the base, no extra cost is incurred in deploying the new node. Other small, mote-based robots are discussed in [19] and [20].

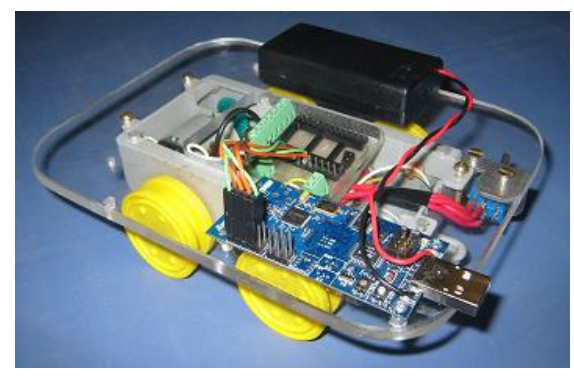

Fig. 6. Tmote-sky controlled robot used for network repair.

\section{B. Harnessing RSSI}

The received signal strength indicator (RSSI) is a measurement inherent to many commercially available transceivers that provides information regarding the RF strength associated with a received message. It is also used for clear channel assessment in some MAC protocols [21]. Strictly speaking, RSSI is not a measurement of how good a communication link is. For such measurements it is best to consult a link quality indicator (LQI). There is however rough correlation between the two and as LQI is not as commonly found on transceivers, the focus of the proposed work will default to RSSI.

RSSI is often used for localization by correlating RSSI measurements to distance [22][23][24][25]. However, RSSI is varying and as such a distance estimate can only be trusted with a certain degree of confidence [26]. This is particularly true as environmental conditions change. While bad for localization, it is precisely this variation that we propose to leverage for network repair.

Using RSSI as a measure of connectivity will allow the robotic system to be flexible, adjusting to the current environmental conditions. If deployment is done on a foggy day, nodes will be placed more compactly than on a clear day since the same RSSI measurement will correlate with a shorter distance. This feature is also inherent to the method implemented in [15]. The advantage of using the RSSI technique however is that it does not require multiple messages and allows for improved controllability without additional sensing. This is a result of the continuity of the RSSI measurement rather than the binary "received" or "not received" message method. This allows the robot to know roughly where it is in relation to its maximum range providing feedback for control.

\section{EXPERIMENTATION}

Here three simple proof of concept experiments are presented on a small, range limited system. First a simple experiment is performed to show variation in RSSI under different physical conditions. Next RSSI navigation is demonstrated by having a robot circle a node by applying simple rules. Lastly, repair of a small network with a single hop disconnectivity is performed.

\section{A. RSSI Variation}

In this experiment a single tmote-sky module emits a beacon signal with reduced transmission power. A robot picks up this signal and moves away until it has exceeded a pre-determined RSSI limit, in this case $-75 \mathrm{dBm}$. The RSSI reading is actually averaged over 2 seconds. Without using an average the robot would hit small momentary dead zones, resulting in stop and go behavior. This is done for three scenarios. First in an open lab area, then repeated in the same area with the range physically attenuated by placing the beacon in a metal box. This is done to mimic an obstruction. The first experiment is then repeated in another lab to demonstrate variation with location. Each scenario is run 15 times. 
TABLE I

DISTANCES TRAVELED FOR THE 3 CASES AVERAGED OVER 15 TRIALS DEMONSTRATING RSSI VARIATION DUE TO ENVIRONMENTAL CHANGES

\begin{tabular}{|l|c|c|}
\hline Location & $\begin{array}{c}\text { Mean Distance } \\
\text { Traveled }\end{array}$ & $\begin{array}{c}\text { Largest Deviation } \\
\text { from Mean }\end{array}$ \\
\hline Lab A & $190 \mathrm{~cm}$ & $41 \mathrm{~cm}$ \\
\hline Lab A, Enclosed & $59 \mathrm{~cm}$ & $8 \mathrm{~cm}$ \\
\hline Lab B & $148 \mathrm{~cm}$ & $31 \mathrm{~cm}$ \\
\hline
\end{tabular}

The averages of the runs are shown in table I and an image is shown in Fig. 7 for comparison. These results clearly demonstrate some of the attenuation of communication in varying environments. It is interesting to note that there were some outliers in the range measurements. In lab A the robot traveled $231 \mathrm{~cm}$, over $\frac{1}{4} \mathrm{~m}$ further than the average. The largest deviation from average in each experiment is also reported in table I. Also if the robot was slightly angled with respect to the beacon, distances as high as $4 \mathrm{~m}$ could be attained. This reinforces the importance of the role that orientation plays in terms of communication range.

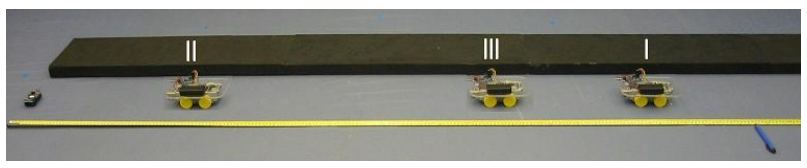

Fig. 7. Comparison of distances travelled for the 3 cases I) lab A II) lab A, enclosed III) lab B

\section{B. RSSI Navigation}

The goal here is to demonstrate navigation using only RSSI. If navigation were based on message type, once out of range the robot would be lost without some sort of localization system. Using RSSI and trying to maintain a given range a robot can move out of the desired range and find its way back depending on whether the signal got lower or higher after each movement decision. The algorithm used is presented in Fig. 8 and a time lapse picture of the robot circling a node while applying these rules is shown in Fig. 9.

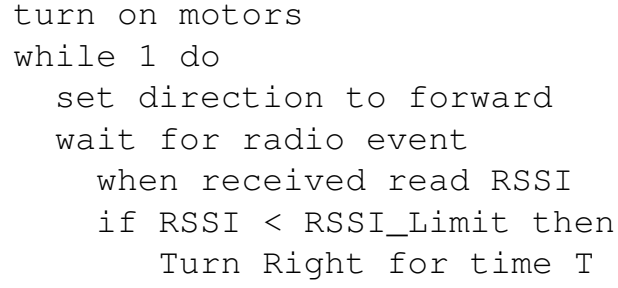

Fig. 8. Control algorithm for basic robot RSSI navigation

Although Fig. 9 shows a good elliptical path, it is necessary to mention that such good results are not always observable. At the lower transmission powers the robot could at times be observed to turn inward prematurely, forming localized loops on one end of the stationary node or sometimes actually colliding with it. This is in part due to the

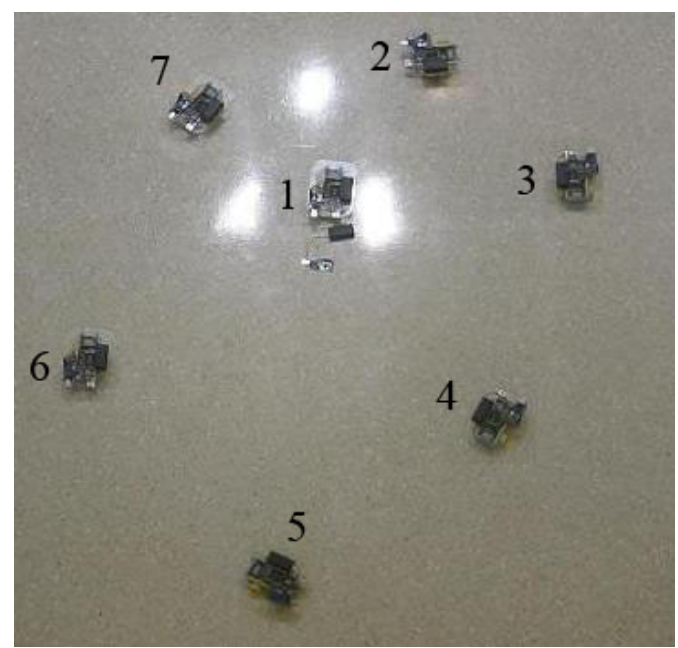

Fig. 9. Time lapse photo of RSSI navigation taken at time steps of approximately $2 \mathrm{~s}$. From the stationary mote the robot moves out and then clockwise around it, staying within the specified RSSI range

orientation of the two nodes which is changing constantly. As shown in experiment 1 , this orientation change can result in great variation. Unfortunately, currently constrained to an indoor environment, a larger scale and higher power test cannot be conducted which would likely not have this same effect due to a farther reaching signal. Also, note that the turn is set to a static angle and in this example only turns right. Tests have been conducted using both left and right turns but no real difference is observable at this scale other than an occasional wiggle. In future implementations, it would likely be appropriate to scale the angle of the turn to the power of the received signal or desired distance. The further away from the desired range, the greater the turn angle. This essentially would implement proportional control. Keeping track of the previously received value and direction would then add derivative control component to the system.

\section{Network Repair}

A simple two node network was constructed with a single hop disconnection. The robot periodically pings to find a node and once found continues to ping circling it until it receives an answer from a second node. The mobile node then becomes the link in the network. Here node ' $S$ ' mimics receiving an event by displaying a count to the LEDS and broadcasting it to the network. When the mobile node repairs the network it then propagates the state to node ' $D$ '. This was successfully accomplished and is demonstrated in the photo series of Fig. 10. As shown in Fig. 2 many of the disconnections could be repaired with a single node as demonstrated here. The robot did not stop directly between the two nodes, rather it stopped when he could hear both. Not only does this still repair the network but provides greater radio coverage of the area but less overlap. This is adjustable however by reducing the range at which the robot travels from a node. It is evident that this was done in Fig. 10 as the experiment was performed in a hallway. 


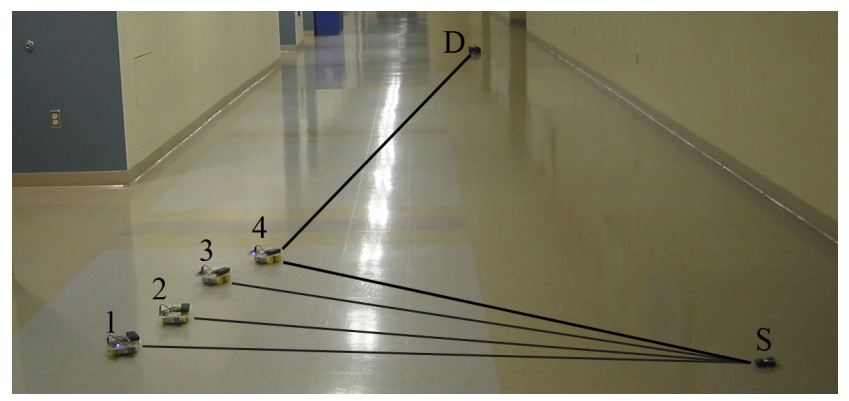

Fig. 10. Time lapse photo of network repair. At time step 4 the message from the source node, $\mathrm{S}$, is routed to the destination node, $\mathrm{D}$.

\section{CONCLUSION}

This paper has introduced a low cost and adaptive method of repair in large area wireless sensor networks using mobile robots. The adaptability gained by using RSSI measurements allows the system to account for variation in communication due to environmental and RF patterns of nodes. This is demonstrated through experimentation and repair of a single hop network. What keeps the system low cost is that it requires no additional sensing capability. This is particularly beneficial in environments where the robot is unlikely to be retrieved.

Future research will be extended to include cooperative, multi-robot systems. Utilizing a mobile sensor web, where each node maintains a communication range with its neighbors, will help resolve more complex, multi-hop disconnections. Also, such a sensor web will be viewed as a means of sensor extension for a more capable robot. With this sensory extension a robot will gather a wealth of environmental data and localize on features much more quickly than by traditional, localized search methods. Research continues into evaluating disconnectivity in deployments so as to optimize cost functions.

\section{REFERENCES}

[1] C. Hartung, R. Han, C. Seielstad, and S. Holbrook, "Firewxnet: A multi-tiered protable wireless system for monitoring weather conditions in wildland fire environments," in 4th International Conference on Mobile Systems, Applications and Services, June 2006, pp. $28-41$.

[2] D. M. Doolin and N. Sitar, "Wireless sensors for wildfire monitoring," in SPIE Symposium on Smart Structures and Materials 2005, San Diego, CA, March 2005, pp. 57-65.

[3] K. Martinez, R. Ong, and J. Hart, "Glacsweb: a sensor network for hostile environments," in IEEE SECON 2004, October 2004, pp. 8187.

[4] K. A. Delin, R. P. Harvey, N. A. Chabot, S. P. Jackson, M. Adams, D. W. Johnson, and J. T. Britton, "Sensor web in antarctica: developing an intelligent, autonomous platform for locating biological flourishes in cryogenic environments," in 34th Lunar and Planetary Science Conference, 2003.

[5] G. Werner-Allen, K. Lorincz, M. Ruiz, O. Marcillo, J. Johnson, J. Lees, and M. Welsh, "Deploying a wireless sensor network on an active volcano," IEEE Internet Computing, vol. 10, no. 2, pp. 18-25, March-April 2006.

[6] A. Fanimokun and J. Frolik, "Effects of natural propagation environments on wireless sensor network coverage area," in Proceedings of the 35th Southeastern Symposium on System Theory, 2003, pp. 16-20.
[7] G. Anastasi, A. Falchi, A. Passarella, M. Conti, and E. Gregori, "Performance measurements of motes sensor networks," in Proceedings of the 7th ACM International Symposium on Modeling, Analysis and Simulation of Wireless and Mobile Systems, Venice, Italy, 2004, pp. 174-181.

[8] P. Corke, S. Hrabar, R. Peterson, D. Rus, S. Saripalli, and G. Sukhatme, "Autonomous deployment and repair of a sensor network using an unmanned aerial vehicle," in Proceedings of the 2004 IEEE International Conference on Robotics and Automation, New Orleans, LA, April 2004.

[9] G. Zhou, T. He, S. Krishnamurthy, and J. A. Stankovic, "Impact of radio irregularity on wireless sensor networks," in Proceedings of the 2nd International Conference On Mobile Systems, Applications and Services, Boston, MA, 2004, pp. 125-138.

[10] D. Lymberopoulos, Q. Lindsey, and A. Savvides, "An empirical characterization of radio signal strength variability in 3-d ieee 802.15.4 networks using monopole antennas," in EWSN 06, 2006, pp. 326-341.

[11] D. Ganesan, B. Krishnamachari, A. Woo, D. Culler, D. Estrin, and S. Wicker, "Complex behavior at scale: An experimental study of low-power wireless sensor networks," UCLA/CSD-TR, Tech. Rep. 020013, 2002.

[12] M. Franceschetti, L. Booth, M. Cook, R. Meester, and J. Bruck, Journal of Statistical Physics, vol. 118, no. 3/4, pp. 721-234, 2005.

[13] L. Parker, B. Kannan, X. Fu, and Y. Tang, "Heterogeneous mobile sensor net deployment using robot herding and line-of-sight formations," in Proceedings of the International Conference on Intelligent Robots and Systems, vol. 3, October 2003, pp. 2488-2493.

[14] A. Howard, M. Mataric, and G. S. Sukhatme, "Mobile sensor network deployment using potential fields: A distributed, scalable solution to the area coverage problem," in Proceedings of the 6th International Symposium on Distributed Autonmous Robotics Systems, Fukuoka, Japan, June 2002.

[15] D. O. Popa, H. E. Stephanou, C. Helm, and A. C. Sanderson, "Robotic deployment of sensor networks using potential fields," in Proceedings of the 2004 IEEE International Conference on Robotics and Automation, vol. 1, New Orleans, LA, April 2004, pp. 642-647.

[16] M. A. Batalin and G. S. Sukhatme, "Coverage, exploration and deployment by a mobile robot and communication network," Telecommunication Systems, Special Issue on Wireless Sensor Networks, vol. 26, no. 2, pp. 181-196, 2004.

[17] J. M. Galeotti, "The evbot: A small autonomous mobile robot for the study of evolutionayr algortihms in distributed robotics," Master's thesis, North Carolina State University, 2002.

[18] Moteiv, Tmote-Sky Datasheet, Moteiv, 2006.

[19] S. Bergbriter and K. S. J. Pister, "Cotsbots: An off-the-shelf platform for distributed robotics," in Proceedings of IEEE/RSJ International Conference on Intelligent Robots and Systems, October 2003, pp. 2731.

[20] K. Dantu, M. Rahimi, H. Shah, S. Babel, A. Dhariwal, and G. S. Sukhatme, "Robomote: Enabling mobility in sensor networks," in Fourth International Symposium on Information Processing in Sensor Networks, April 2005, pp. 404-409.

[21] Chipcon, CC2420 Datasheet, Texas Instruments, 2006.

[22] M. Terwilliger, A. Gupta, V. Bhuse, Z. H. Kamal, and M. A. Salahuddin, "A localization system using wireless network sensors: A comparison of two techniques," in Proceedings of the First Workshop on Positioning, Navagation, and Communication, Hanover, Germany, March 2004.

[23] K. Lorincz and M. Welsh, "Motetrack: A robust, decentralized approach to rf-based location tracking," in Proceedings of the International Workshop on Location and Context-Awareness, May 2005.

[24] C. Alippi and G. Vanini, "A rssi-based and calibrated centralized localization technique for wireless sensor networks," in Proceedings of the Fourth Annual IEEE International Conference on Pervasive Computing and Communications Workshops, 2006.

[25] M. Sichitiu, V. Ramadurai, and P. Peddabachagari, "Simple algorithm for outdoor localization of wireless sensor networks with inaccurate range measurements," in Proceedings of the 2003 International Conference on Wireless Networks, Las Vegas, NV, June 2003, pp. 300-305.

[26] V. Ramaduari and M. Sichitiu, "Localization in wireless sensor networks: A probabilistic approach," in Proceedings of the 2003 International Conference on Wireless Networks, Las Vegas, NV, June 2003, pp. 275-281. 